\title{
Quality of life in patients with age-related macular degeneration: results from the VISION study
}

\begin{abstract}
Purpose To assess the impact of treatment with pegaptanib sodium vs usual care on vision-related quality of life (VRQoL) in patients with age-related macular degeneration (AMD).
\end{abstract}

Methods VRQoL was a secondary end point in the trial, a prospective, randomized, doublemasked, multicentre, dose-ranging study. Three doses of pegaptanib $(0.3,1$, and $3 \mathrm{mg})$ were compared with usual care with respect to changes in VRQoL as indicated by the 25-item National Eye Institute Visual Function Questionnaire (NEI-VFQ 25), administered at baseline and weeks 30 and 54 . Four of the NEIVFQ 25 domains were prospectively designated as primary: near vision, distance vision, role limitations, and dependency. Between-group differences were assessed using an analysis of covariance model with age, gender, and baseline score as covariates. Results NEI-VFQ 25 data were available for 569 subjects. At week 54, improvements in the distance vision and role limitations domains were greater in pegaptanib than usual care arms. No substantial increase in ocular pain was noted in pegaptanib-treated patients. No clear superiority of any particular dosage strength of pegaptanib was demonstrated, and no significant differences or trends favoured usual care on any domain score or the NEIVFQ 25 composite score. The greatest VRQoL benefit was seen in responders (lost $<3$ lines) to treatment.

Conclusion The VISION trial provided evidence of trends in quality-of-life benefit associated with effective treatment of AMD using pegaptanib. Treatment with pegaptanib is expected to contribute significantly to VRQoL improvement for responder patients.
A Leys', G Zlateva², SN Shah² and M Patel², for the VEGF Inhibition Study in Ocular Neovascularization (VISION) Clinical Trial Group

Eye (2008) 22, 792-798; doi:10.1038/sj.eye.6702900; published online 22 June 2007

Keywords: age-related macular degeneration; pegaptanib sodium; quality of life

\section{Introduction}

The progressive deterioration of central vision associated with age-related macular degeneration (AMD) profoundly impacts vision-related quality of life (VRQoL). AMDrelated visual acuity loss has been linked with reductions in patient quality of life, an increased need for assistance with activities of daily living, and emotional distress and depression. ${ }^{1-6}$ Moreover, while vision loss in general leads to an increased risk of injury owing to falls, ${ }^{7-9}$ late AMD in particular has been associated with a $70 \%$ increased risk of falling two or more times. ${ }^{10}$ Despite these negative consequences and in spite of the fact that AMD is the leading cause of irreversible vision loss among individuals over 50 years of age in the Western world, ${ }^{11}$ clinicians and community members greatly underestimate the impact of AMD on quality of life. ${ }^{12}$

Given the strong correlation between decreased visual acuity and diminished VRQoL, effective treatment of AMD leading to stabilization or improvement in vision may positively affect patient's quality of life.

Pegaptanib sodium is the first pharmacological treatment indicated for subfoveal AMD and the first approved therapy that targets the underlying pathological process common to all subtypes and classifications of neovascular AMD. ${ }^{13}$ The VEGF Inhibition Study in Ocular Neovascularization (VISION) clinical trial 
found that pegaptanib provided a statistically significant and clinically meaningful benefit to a broad spectrum of AMD patients over both 1 and 2 years. ${ }^{14,15}$ Although the VISION trial, comprised of two identical studies, and was designed principally to establish the safe and efficacious dose of pegaptanib injections (compared to sham control - usual care), patient-reported VRQoL was a secondary end point assessed in one study. The purpose of this investigation was to evaluate the impact of pegaptanib treatment vs usual care on VRQoL in patients with AMD, analysing data from the VISION trial. We hypothesized that improved or stabilized vision would be associated with improved or stabilized VRQoL.

\section{Materials and methods}

\section{Data source}

Details of the VISION trial have been published. ${ }^{14-16}$ In brief, two concurrent, prospective, randomized, double-blind, sham-controlled, dose-ranging pivotal studies enrolled 1208 patients at 117 sites in the United States, Canada, Europe, Israel, Australia, and South America. Eligibility criteria included (1) age $\geqslant 50$ years, (2) presence of subfoveal sites of choroidal neovascularization (CNV) secondary to AMD, (3) best-corrected study eye visual acuity range of 20/40 to $20 / 320$, and (4) best-corrected fellow eye visual acuity of 20/800 or better. Patients with all angiographic subtypes were enrolled. Lesions could not exceed 12 total disc areas (including blood, scar or atrophy, and neovascularization), of which at least $50 \%$ had to be active CNV. No more than $50 \%$ of the lesion could be a subretinal haemorrhage.

Patients were randomly assigned to receive intravitreous injections of pegaptanib at $0.3,1$, or $3 \mathrm{mg}$, or to receive sham injection (usual care) into one eye, the study eye, every 6 weeks for 48 weeks. The use of photodynamic therapy with verteporfin was allowed in patients with predominantly classic lesions in all study arms at the discretion of the treating ophthalmologist. The injection protocol for patients receiving sham injections was identical to that used in the treatment groups, with the exception of scleral penetration.

\section{Vision-related quality of life}

VRQoL, which was assessed in one of the two studies that comprised the VISION trial, was measured by the 25-item National Eye Institute Visual Function Questionnaire (NEI-VFQ 25), a reliable and validated measurement tool. ${ }^{17}$ The questionnaire was either self-administered by patients or administered by trained
Table 1 National Eye Institute Visual Function Questionnaire 25 domains

\begin{tabular}{lc}
\hline Domain & Number of items \\
\hline Near vision & 3 \\
Distance vision & 3 \\
Role limitations & 2 \\
Dependency & 3 \\
Mental health & 4 \\
Ocular pain & 2 \\
Social functioning & 2 \\
Driving & 2 \\
Colour vision & 1 \\
Peripheral vision & 1 \\
General vision & 1 \\
General health & 1 \\
Composite (overall VRQoL) & 25 \\
\hline
\end{tabular}

VRQoL, vision-related quality of life.

interviewers in a face-to-face interview format. At the time the VISION trial was initiated, only the US English-language version had been validated. ${ }^{17}$ Therefore, only those patients enrolled in the United States and Canada completed the assessment.

The NEI-VFQ 25 measures visual disabilities and their impact on daily functioning, and has been used in a variety of vision-impaired patient populations. ${ }^{18-21}$ The instrument consists of 12 domains plus a composite score of overall VRQoL (Table 1).

In this study, four domains were designated a priori as primary domains, that is, those most likely to be characteristic of AMD disease progression. These included near vision, distance vision, role limitations, and dependency. Domain and composite scores can range from 0 (worst-possible VRQoL) to 100 (best-possible VRQoL). The NEI-VFQ 25, which usually takes about $10 \mathrm{~min}$ to complete, was administered at baseline and weeks 30 and 54 . The questionnaire was either self-administered by patients or administered by trained interviewers in a face-to-face interview format; questionnaires were administered before injection.

\section{Data analyses}

The end point of interest in this analysis, changes from baseline to week 54 in the domain and composite scores of the NEI-VFQ 25, was a secondary end point in the VISION trial. By design, the VISION trial was powered to detect differences between treatments in the primary study end point (loss of $<3$ lines of visual acuity from baseline to week 54), but not with respect to NEI-VFQ 25 domain scores.

Changes in VRQoL were compared between each, the pegaptanib dosage group and the usual care group, 
using analyses of covariance (ANCOVA) models controlling for age, gender, and baseline domain score. Any positive differences between the pegaptanib group and the usual care group indicated a treatment benefit of pegaptanib. We focused primarily on differences in the four primary domains of interest (near vision, distance vision, role limitations, and dependency). An intentionto-treat analysis was used such that patients completing one or more questions on any domain at baseline were included in the analysis. Except for the driving domain, missing observations at follow-up were handled using a last observation carried forward approach (from week 30 or baseline). Patients missing a baseline score for a domain were excluded from the analysis for that domain, although responses for other domains with sufficient responses to yield a score were included in the analysis. $\alpha$ was set at 0.05 for all comparisons.

A second set of analyses compared the VRQoL of treatment responders $v s$ treatment non-responders as defined by the primary end point of the VISION trial-lines of vision lost. ${ }^{14}$ The three pegaptanib dosage groups and the usual care group were combined to increase the sample size and hence the power to detect differences between responders and non-responders in mean NEI-VFQ domain scores. Responders were defined as patients losing $<3$ lines of vision over the 54-week study period, regardless of therapy. Non-responders were those losing $\geqslant 3$ lines of vision over 54 weeks, regardless of therapy. Follow-up NEI-VFQ 25 domain and composite scores for responders vs non-responders were compared using ANCOVA models controlling for age, gender, baseline domain score, and the visual acuity difference between the study (treated) eye and the fellow (untreated) eye. $\alpha$ was set at 0.05 for all between-group comparisons.

\section{Results}

A total of 578 patients were enrolled in the United States or Canada and received at least one on-study treatment (safety population: 144 for $0.3 \mathrm{mg} ; 146$ for $1 \mathrm{mg} ; 143$ for $3 \mathrm{mg}$; and 145 for usual care). Patients in the four treatment groups were similar with regard to gender, race, age, and baseline visual acuity (Table 2).

NEI-VFQ 25 data were available for 569/578 (98.4\%) patients and were the focus of this analysis. Patients were equally distributed among the treatment groups (141 for $0.3 \mathrm{mg}$; 144 for $1 \mathrm{mg}$; 141 for $3 \mathrm{mg}$; and 143 for usual care). For the four primary domains, the actual sample sizes were less than half of those required to detect a five-point difference over time between any two groups (Table 3). ${ }^{17}$

Outcomes of VRQoL assessments depend on whether the subject has the worse or better eye treated, with the strongest relationship between VRQoL and visual acuity in the better-seeing eye, and little or no relationship between VRQoL and the worse-seeing eye. ${ }^{22,23}$ By chance, all three doses with pegaptanib had a larger proportion of patients whose worse eye was treated, whereas the usual care group had the largest proportion of patients whose better eye was treated (Table 4). We considered the better/worse eye observation to be important in analyses of within-group differences from baseline to week 54 (eg, 0.3-0.3 mg), but not in betweengroup analyses (eg, $0.3 \mathrm{mg}$-usual care). At baseline,

Table 2 Patient characteristics at baseline, safety population

\begin{tabular}{|c|c|c|c|c|}
\hline \multirow[t]{2}{*}{ Characteristic } & \multicolumn{3}{|c|}{ Pegaptanib } & \multirow[t]{2}{*}{ Usual care, $\mathrm{N}=145$} \\
\hline & $0.3 m g, \mathrm{~N}=144$ & $1 m g, \mathrm{~N}=146$ & $3 m g, \mathrm{~N}=143$ & \\
\hline \multicolumn{5}{|l|}{ Gender, $n(\%)$} \\
\hline Male & $64(44)$ & $68(47)$ & $45(31)$ & $73(43)$ \\
\hline Female & $80(56)$ & $78(53)$ & $98(69)$ & $82(57)$ \\
\hline \multicolumn{5}{|l|}{ Race, $n(\%)$} \\
\hline White & $140(97)$ & $143(98)$ & $141(99)$ & $140(97)$ \\
\hline Other & $4(3)$ & $3(2)$ & $2(1)$ & $5(3)$ \\
\hline \multicolumn{5}{|l|}{ Age, years } \\
\hline Mean \pm SD & $78.0 \pm 7.5$ & $76.5 \pm 6.8$ & $77.1 \pm 7.5$ & $76.7 \pm 6.6$ \\
\hline Range & $58-92$ & $52-92$ & $56-97$ & $55-89$ \\
\hline \multicolumn{5}{|c|}{ Visual acuity, study eye (ETDRS) } \\
\hline Mean \pm SD & $52.5 \pm 12.8$ & $50.5 \pm 12.3$ & $52.1 \pm 12.7$ & $54.0 \pm 11.0^{\mathrm{a}}$ \\
\hline Range & $23-74$ & $19-73$ & $14-73$ & $27-74$ \\
\hline
\end{tabular}

Abbreviations: ETDRS, Early Treatment Diabetic Retinopathy Study; SD, standard deviation.

${ }^{\mathrm{a}}$ Missing data for one subject. 
scores for the primary domains of interest were similar across treatment groups (Table 5).

\section{Changes in VRQoL from baseline to week 54}

Changes in scores on the four primary domains of the NEI-VFQ 25 are displayed in Figure 1. As Figure 1 shows, mean scores on the distance vision and role limitations domains were consistently higher in the active treatment groups than in the usual care group. For distance vision, least square (LS) mean score differences between pegaptanib and usual care were similar across pegaptanib groups (LS diff $=4.30, P=0.059$ for $0.3 \mathrm{mg}$; LS diff $=4.63, P=0.041$ for $1 \mathrm{mg}$; and LS diff $=5.83$, $P=0.011$ for $3 \mathrm{mg}$ ). For role limitations, while all active treatment groups showed improvements compared to

Table 3 Required $v s$ actual sample sizes for primary domains

\begin{tabular}{lcc}
\hline Domain & \multicolumn{2}{c}{ Sample size per group } \\
\cline { 2 - 3 } & Required $^{\mathrm{a}}$ & Actual \\
\hline Near vision & 338 & $141-144$ \\
Distance vision & 338 & $141-144$ \\
Role limitations & 338 & $141-144$ \\
Dependency & 315 & $141-144$
\end{tabular}

aSample sizes required per group to detect a five-point difference over time between two experimental groups for the NEI-VFQ 25. Estimates assume $\alpha=0.05$ for a two-tailed $t$-test with power $=80 \%$ and an intertemporal correlation between scores $=0.60 .{ }^{17}$

Table 4 Patients with better and worse eye treated at baseline $\mathrm{a}^{\mathrm{a}}$

\begin{tabular}{lcccccc}
\hline Treatment group & All, N & \multicolumn{2}{c}{ Better eye treated } & & \multicolumn{2}{c}{ Worse eye treated } \\
\cline { 3 - 4 } \cline { 7 - 8 } & & $\mathrm{N}$ & $\%$ & & $\mathrm{~N}$ & $\%$ \\
\hline 0.3-mg & 141 & 57 & 40.4 & & 84 & 59.6 \\
1-mg & 142 & 57 & 40.1 & & 85 & 59.9 \\
3-mg & 141 & 47 & 34.8 & & 92 & 65.2 \\
Usual care & 142 & 64 & 45.1 & & 78 & 54.9 \\
\hline
\end{tabular}

aix patients had eyes with the same visual acuity and were classified as 'better eye treated'. usual care, only the 3-mg group showed a statistically significant improvement (LS diff $=7.66, P=0.007$ ), although the difference for the $0.3-\mathrm{mg}$ group approached significance (LS diff $=5.35, P=0.060$ ). Changes in the near vision and dependency domains were in different directions across the three pegaptanib doses and did not reach statistical significance.

Among the non-primary domains, differences between patients in ocular pain treated with any dose of pegaptanib and those receiving usual care were not statistically significant, confirming that pegaptanib does not substantially increase ocular pain (LS diff for $0.3,1$, and $3 \mathrm{mg}$ is $-2.55,-2.61$, and -1.74 , respectively; $P>0.10$ for each). Statistically significant differences between certain pegaptanib groups and usual care were present for some non-primary domains. For colour vision, both the $3-\mathrm{mg}$ (LS diff $=5.88, P=0.007$ ) and $0.3-\mathrm{mg}$ (LS diff $=4.64, P=0.032$ ) groups showed improvement compared to usual care. For both social functioning and peripheral vision, the 3-mg group showed improvement

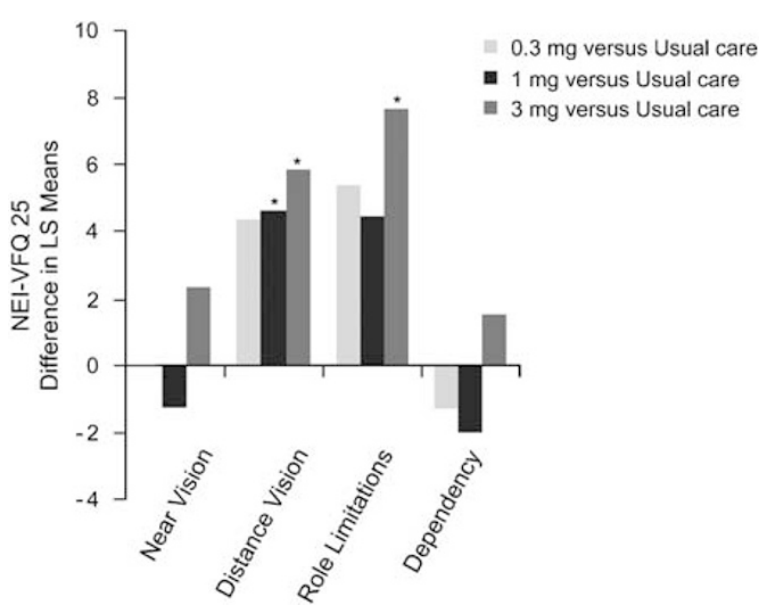

Figure 1 Changes in NEI-VFQ 25 scores for primary domains from baseline to week 54 . ${ }^{*} P<0.05$. LS, least squares; $N=568$ for near vision and distance vision domains; $N=569$ for role limitations and dependency domains. The difference in LS means on the near vision domain for $0.3 \mathrm{mg}$ vs usual care $=-0.02$, which appears to be 0 in the figure.

Table 5 Baseline NEI-VFQ 25 scores, primary domains ${ }^{\text {a }}$

\begin{tabular}{lcccc}
\hline Domain & \multicolumn{3}{c}{ Pegaptanib } & Usual care, $\mathrm{N}=143$ \\
\cline { 2 - 4 } & $0.3 m g, \mathrm{~N}=141$ & $1 m g, \mathrm{~N}=144$ & $3 m g, \mathrm{~N}=141$ & \\
\hline Near vision & $55.1 \pm 24.8$ & $55.1 \pm 24.4$ & $56.5 \pm 23.5$ & $52.1 \pm 25.7$ \\
Distance vision & $65.1 \pm 22.9$ & $65.0 \pm 23.6$ & $64.1 \pm 22.7$ & $62.4 \pm 24.8$ \\
Role limitations & $62.2 \pm 29.2$ & $65.2 \pm 28.9$ & $65.5 \pm 28.2$ & $61.0 \pm 30.5$ \\
Dependency & $73.6 \pm 29.8$ & $78.2 \pm 26.2$ & $75.5 \pm 26.6$ & $71.1 \pm 30.3^{\mathrm{b}}$ \\
\hline
\end{tabular}

${ }^{\mathrm{a}}$ Mean $\pm \mathrm{SD}$.

${ }^{\mathrm{b}}$ Missing data for one patient. 
compared to usual care (LS diff $=6.16, P=0.009$ for social functioning; LS diff $=5.23, P=0.030$ for peripheral vision).

The presence of ceiling and floor effects was assessed at baseline because improvement or deterioration in a domain cannot be detected in those entering a study with either the best- or the worst-possible score. Several domain scores exhibited ceiling effects at baseline. Dependency, ocular pain, social functioning, colour vision, and peripheral vision all had more than one-third of respondents score at the ceiling (highest possible score). One domain score, driving, exhibited a floor effect at baseline ( $25 \%$ of patients recorded the lowest possible score).

\section{VRQoL by response to treatment at week 54}

Comparisons of week 54 NEI-VFQ 25 domain scores by response to treatment (any pegaptanib dose or usual care) are displayed in Figure 2. As shown in Figure 2, responders had significantly higher follow-up scores on all four primary domains (near vision, distance vision, role limitations, and dependency) compared to non-responders (all $P$-values $<0.05$ ). Compared to non-responders, responders also had significantly higher follow-up scores on the mental health, general vision, social functioning, and driving domains, as well as the composite score (all $P$-values $<0.05$ ). Most of the differences between the responder groups exceeded a magnitude of five points. Consistent with the clinical profile of AMD, there was little impairment in peripheral vision or ocular pain at week 54, but substantial impairment in near vision, distance vision, and driving.

\section{Discussion}

Pegaptanib demonstrated the ability to preserve or improve vision in more than one-third of AMD patients and halted progression of vision loss in two-thirds of patients in the first year of the VISION trial. ${ }^{14} \mathrm{We}$ hypothesized that this clinical benefit would be associated with stability or improvement in VRQoL, and found trends in VRQoL benefit associated with pegaptanib therapy in a subgroup of patients in whom VRQoL was measured. In particular, improvements in the distance vision and role limitations domains were greater in pegaptanib than in usual care arms, while no substantial increase in ocular pain was noted in pegaptanib-treated patients compared to usual care. No clear superiority of any particular dosage strength of pegaptanib was demonstrated, and no significant differences or trends favoured usual care on any domain score or the composite score of the NEI-VFQ 25.

Disease characteristics and ceiling effects may explain why improvements in pegaptanib groups relative to usual care in distance vision and role limitations were not paralleled by positive changes in near vision or dependency, respectively (Figure 1). AMD patients experience loss of central vision, and it seems likely that the improvement in distance but not near vision reflects stabilization or improvement in less affected, non-central vision. The observation that changes in the role limitations and dependency domains were in opposite directions may reflect the fact that $34 \%$ of patients had the highest possible score on the dependency domain at baseline, which severely limited our ability to detect improvement.

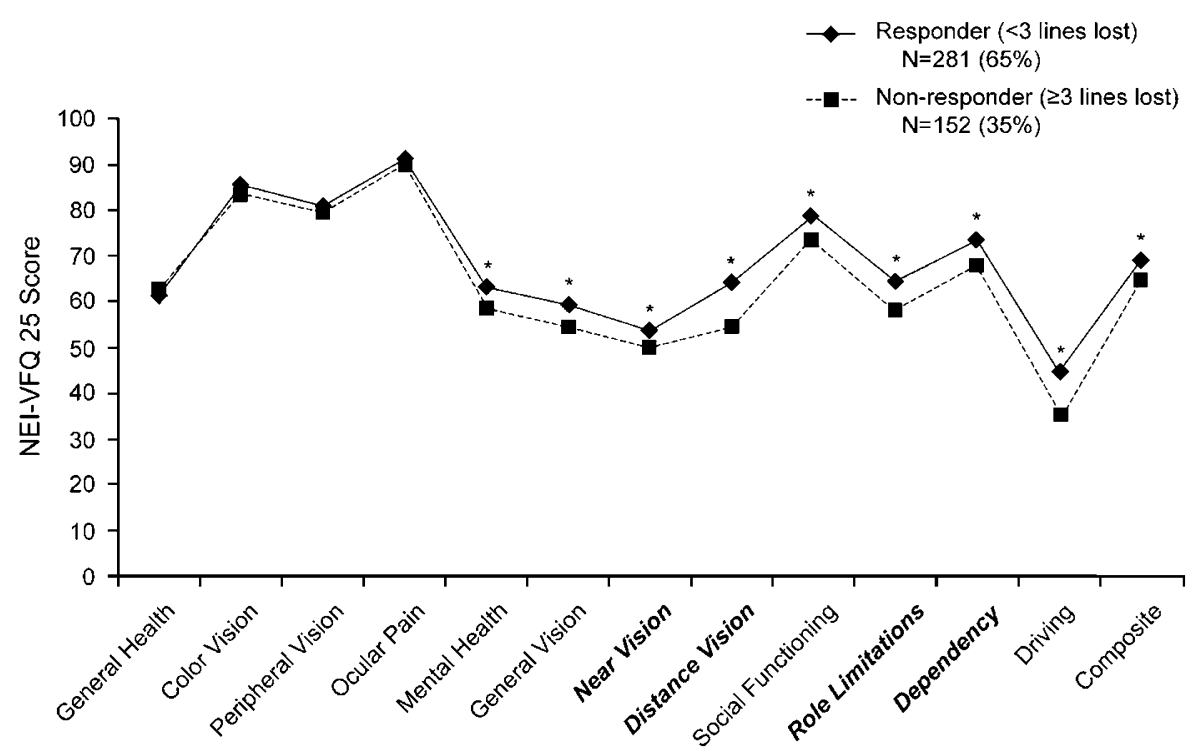

Figure 2 VRQoL by responder status at week 54 . The four primary domains are in italics. ${ }^{*} P<0.05$; analysis of covariance model with age, gender, baseline domain score, and magnitude of difference in baseline visual acuity between eyes as covariates. 
Overall, good vision was associated with good VRQoL outcomes. Responders to any treatment, either one of the pegaptanib dosages or usual care, showed a statistically significant VRQoL benefit compared to non-responders on 8 of the 12 domains, including the 4 primary domains and the composite domain. Because significantly more subjects treated with pegaptanib were responders, ${ }^{14}$ active treatment had a beneficial effect on VRQoL. These data and those of the Submacular Surgery Trials (SST) pilot study suggest that differences of 5-10 points on NEI-VFQ 25 domains are clinically meaningful to patients. $^{24}$

The lack of statistically significant differences between active treatment and usual care for some NEI-VFQ 25 domains may have been due, at least in part, to small sample sizes. As shown in Table 3, actual sample sizes for the primary domains were less than half of those required to detect five-point differences between two groups over time. ${ }^{17}$ The VISION trial was not powered to detect small but potentially meaningful differences in secondary end points, including NEI-VFQ 25 domain scores.

Other factors may have further restricted our ability to detect differences between pegaptanib and usual care. Self-administration of the NEI-VFQ 25 by a substantial number of patients may have impacted the findings if patients were unable to read the questionnaire by themselves; administration of the NEI-VFQ 25 by trained telephone interviewers may be preferable in vision-impaired respondents. ${ }^{25,26}$ In addition, the questionnaire was administered through different modes (self- or interviewer-administered), but the mode of administration was not documented in the VISION study. Previous research ${ }^{27}$ has shown that the two modes of administration may not produce identical results on health-related QoL measures, and any inconsistencies across time periods in this study may have increased variation, making it more difficult to demonstrate statistically significant differences in health-related QoL end points. The ability of the NEI-VFQ 25 to demonstrate prospective changes in vision may have been attenuated, as more than acceptable ceiling effects were noted for the role limitations and dependency domains, and floor effects were seen for several secondary domains. Finally, although NEI-VFQ 25 scores show the highest correlation to the vision status of the better-seeing eye, ${ }^{23}$ the majority of patients enrolled in the VISION trial had the eye with the worst visual acuity treated.

Even given these limitations, results from the VISION trial provide evidence of trends in quality-of-life benefit associated with vision stabilization or improvement following treatment of AMD with pegaptanib. In patients facing a diminished quality of life owing to AMD-related vision loss, a treatment such as pegaptanib that slows disease progression may be associated with maintained or improved VRQoL, especially in responder patients. Early detection and treatment of AMD may have the greatest impact on preserving vision and vision-related functioning.

\section{Acknowledgements}

Editorial support, including contributing to the first draft of this paper, revising the paper based on author feedback, and styling the paper for journal submission, was provided by Dr Jane Murphy of Zola Associates and funded by Pfizer Inc. The VISION study was sponsored by (OSI) Eyetech Inc., New York, NY, USA and Pfizer Inc., New York, NY, USA.

\section{References}

1 Brody BL, Gamst AC, Williams RA, Smith AR, Lau PW, Dolnak D et al. Depression, visual acuity, comorbidity, and disability associated with age-related macular degeneration. Ophthalmology 2001; 108: 1893-1900.

2 DeCarlo DK, Scilley K, Wells J, Owsley C. Driving habits and health-related quality of life in patients with age-related maculopathy. Optom Vis Sci 2003; 80: 207-213.

3 Hazel CA, Petre KL, Armstrong RA, Benson MT, Frost NA. Visual function and subjective quality of life compared in subjects with acquired macular disease. Invest Ophthalmol Vis Sci 2000; 41: 1309-1315.

4 Mangione CM, Gutierrez PR, Lowe G, Orav EJ, Seddon JM. Influence of age-related maculopathy on visual functioning and health-related quality of life. Am J Ophthalmol 1999; 128: $45-53$.

5 Scott IU, Feuer WJ, Jacko JA. Impact of visual function on computer task accuracy and reaction time in a cohort of patients with age-related macular degeneration. Am J Ophthalmol 2002; 133: 350-357.

6 Williams RA, Brody BL, Thomas RG, Kaplan RM, Brown SI. The psychosocial impact of macular degeneration. Arch Ophthalmol 1998; 116: 514-520.

7 Coleman AL, Stone K, Ewing SK, Nevitt M, Cummings S, Cauley JA et al. Higher risk of multiple falls among elderly women who lose visual acuity. Ophthalmology 2004; 111: 857-862.

8 Glynn RJ, Seddon JM, Krug Jr JH, Sahagian CR, Chiavelli ME, Campion EW. Falls in elderly patients with glaucoma. Arch Ophthalmol 1991; 109: 205-210.

9 Ivers RQ, Cumming RG, Mitchell P, Simpson JM, Peduto AJ. Visual risk factors for hip fracture in older people. $J \mathrm{Am}$ Geriatr Soc 2003; 51: 356-363.

10 Ivers RQ, Cumming RG, Mitchell P, Attebo K. Visual impairment and falls in older adults: the Blue Mountains Eye Study. J Am Geriatr Soc 1998; 46: 58-64.

11 Harvey PT. Common eye diseases of elderly people: identifying and treating causes of vision loss. Gerontology 2003; 49: 1-11.

12 Stein JD, Brown MM, Brown GC, Hollands H, Sharma S. Quality of life with macular degeneration: perceptions of 
20 Parrish II RK, Gedde SJ, Scott IU, Feuer WJ, Schiffman JC, Mangione CM et al. Visual function and quality of life among patients with glaucoma. Arch Ophthalmol 1997; 115: 1447-1455.

21 Cahill MT, Banks AD, Stinnett SS, Toth CA. Vision-related quality of life in patients with bilateral severe age-related macular degeneration. Ophthalmology 2005; 112: 152-158.

22 Brown GC, Sharma S, Brown MM, Kistler J. Utility values and age-related macular degeneration. Arch Ophthalmol 2000; 118: 47-51.

23 Deramo VA, Cox TA, Syed AB, Lee PP, Fekrat S. Vision-related quality of life in people with central retinal vein occlusion using the 25-item National Eye Institute Visual Function Questionnaire. Arch Ophthalmol 2003; 121: 1297-1302.

24 Miskala PH, Hawkins BS, Mangione CM, Bass EB, Bressler NM, Dong LM et al. Responsiveness of the National Eye Institute Visual Function Questionnaire to changes in visual acuity: findings in patients with subfoveal choroidal neovascularization - SST Report No. 1. Arch Ophthalmol 2003; 121: 531-539.

25 Hall TA, McGwin Jr G, Searcey K, Xie A, Hupp SL, Owsley $\mathrm{C}$ et al. Health-related quality of life and psychosocial characteristics of patients with benign essential blepharospasm. Arch Ophthalmol 2006; 124: 116-119.

26 Berdeaux GH, Nordmann JP, Colin E, Arnould B. Vision-related quality of life in patients suffering from age-related macular degeneration. Am J Ophthalmol 2005; 139: 271-279.

27 Weinberger M, Nagle B, Hanlon JT, Samsa GP, Schmader K, Landsman PB et al. Assessing health-related quality of life in elderly outpatients: telephone versus face-to-face administration. Am J Geriatr Soc 1994; 42: 1295-1299. 